\title{
Identification of R-peaks to Detect Ventricular Fibrillation, Ventricular Tachycardia and Atrial Fibrillation
}

\author{
Manjula Kumbar' ${ }^{1}$, ijay Rayar $^{2}$ \\ ${ }^{1}$ IV Semester M.Tech. Student, Dept. of Electronics and Communication Engineering, \\ K.L.E. Dr. M.S.Sheshgiri College of Engineering and Technology, Belagum-590008, Karnataka, India-590018 \\ ${ }^{2}$ Faculty Dept. of Electronics and Communication Engineering, \\ K.L.E. Dr. M.S.Sheshgiri College of Engineering and Technology, Belagum-590008, Karnataka, India-590018
}

\begin{abstract}
Many types of arrhythmias are identified by where they occur in the heart either in the Atria or Ventricles and what happens to the heart rhythm when they occur. Arrhythmias that begin in the atria are called atrial or super ventricular arrhythmias. Arrhythmias that begin in the ventricles are called ventricular arrhythmias. These ventricular arrhythmias begin in ventricles. The rhythm of heart can be measured using ECG signal. In this work, we are detecting R-peaks using Pan and Tompkins algorithm by which we are calculating the heart rate from ECG signal. These heart rate and abnormalities in ECG signal help us to detect VT (Ventricular Tachycardia), AF (Atrial Fibrillation) and VF (Ventricular Fibrillation).This Pan and Tompkins algorithm is implemented using MATLAB.
\end{abstract}

Keywords: ECG, Pan-Tompkins algorithm, R-peaks

\section{Introduction}

Arrhythmia means abnormal beating of heart. In which increase in heart beat is called Tachyarrhythmia and decrease in heart beat is called Brady arrhythmia which leads to heart attack [1]. Arrhythmia is due to abnormal conducting of tissue within the heart, it could be in atria or ventricle. The graphical representation of cardiac movement which is generated by the cardiac muscle is called ECG. The ECG with PQRST peaks are shown in the fig. 1 .

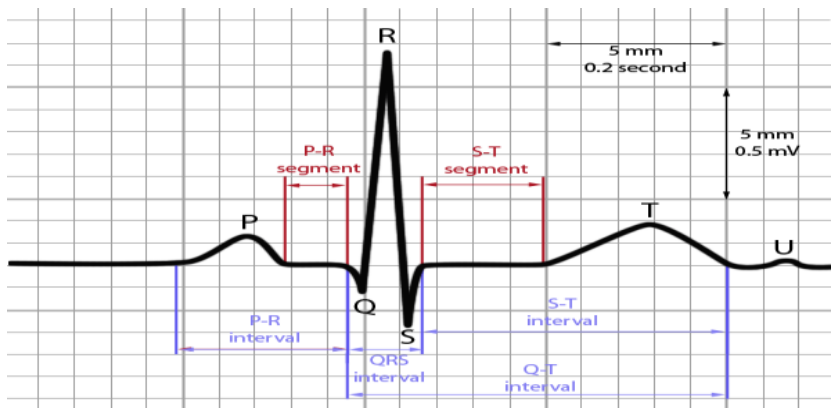

Figure 1: Normal ECG signal with PQRST peaks.

Arrhythmia means abnormal beating of heart. In which increase in heart beat is called Tachyarrhythmia and decrease in heart beat is called Brady arrhythmia which leads to heart attack. Arrhythmia is due to abnormal conducting of tissue within the heart, it could be in atria or ventricle. The above figure. 2 represents where the atria and ventricles are present in the heart [1].

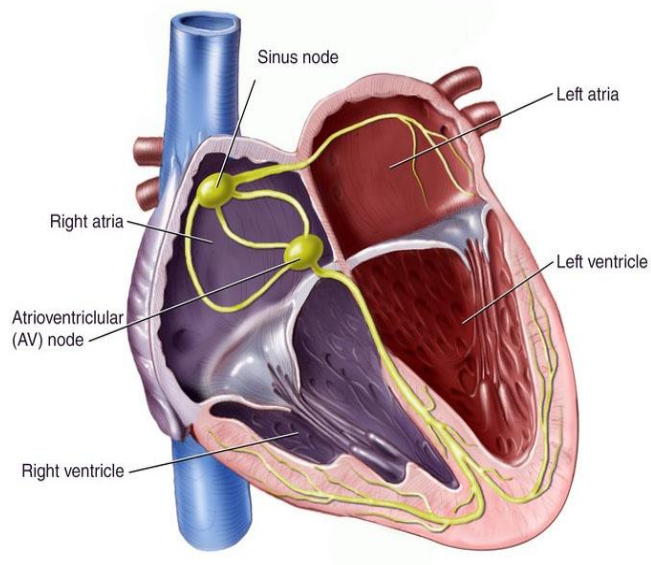

Figure 2: Cardiac Arrhythmia

Main concern in this topic is,

- Atrial Fibrillation (AF)

- Ventricular Tachycardia (VT)

- Ventricular Fibrillation (VF)

Now let's discuss about the mechanism of AF, VT and VF in detail.

- Ventricular Tachycardia:

In Ventricular tachycardia (VT) the heart rate is fast and regular, which occurs in ventricles during the abnormality of the heart. Only for few seconds it may not cause problem, longer duration lead to VT and it can turn into VF. Initially in $7 \%$ of the people with cardiac arrest VT is found. Heart rate reaches up to $150-200 \mathrm{bpm}$.

Definition:

Ventricular tachycardia (VT) is a type of regular and fast heart rate that arises from improper electrical activity in the ventricles of the heart. 


\section{International Journal of Science and Research (IJSR) \\ ISSN (Online): 2319-7064}

Index Copernicus Value (2015): 78.96 Impact Factor (2015): 6.391

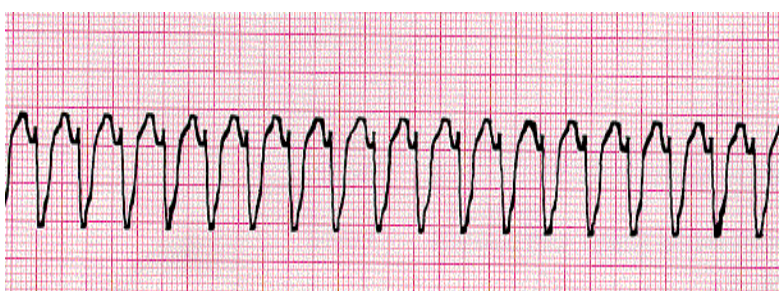

Figure 3: VT detected ECG sample

\section{- Atrial fibrillation:}

In Atrial fibrillation (AF) the main mechanism is re-entry, which takes over SA node and makes the heart beat fast. So in atrial fibrillation and flutter atria beat faster than ventricle and gets impulses after many atrial contractions. AF leads to shortness of breath and chest pain there may be a risk of heart failure and stroke. Heart rate reaches up to $100-150 \mathrm{bpm}$.

\section{Definition:}

Atrial fibrillation, known as AF or A fib, is an irregular and often very fast heart rate.

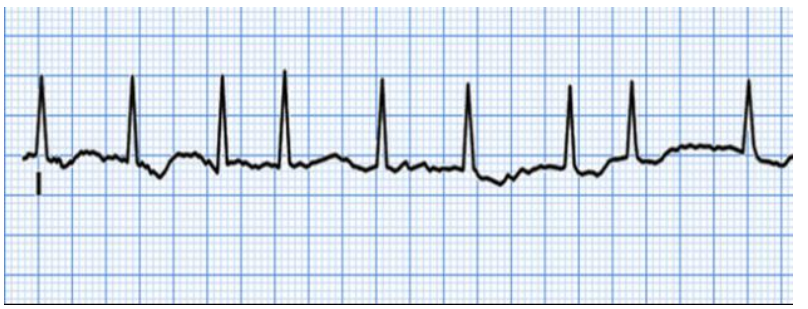

Figure 4: AF detected ECG signal

\section{- Ventricular fibrillation:}

VT, presenting with $300 \mathrm{bpm}$ heart rate is called VF. ECG shows irregular extremely fast, small potential fluctuation in rate. It is fatal condition. As fast beating heart cannot pump blood leads to sudden death. In VF heart rate reaches up to 200-300bpm and above.

\section{Definition:}

An abnormal and irregular heart rhythm in which there are uncoordinated contractions of the lower chambers of the heart.

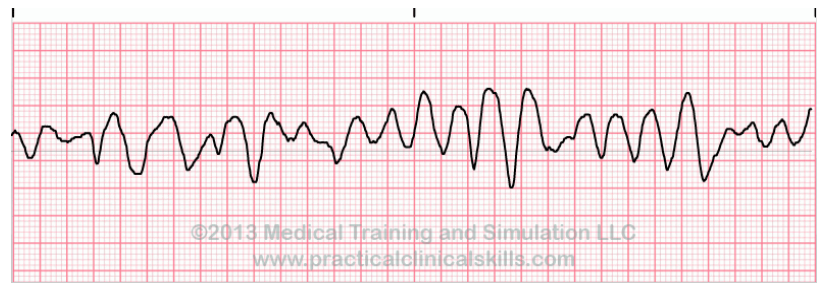

Figure 5: VF detected ECG signal

\section{Methodology}

In this chapter, let's discuss about how the ECG signal processing can be done and how it can be extracted to give input, methodology and algorithm which has been used for the implementation.

\section{Extraction of ECG signal:}

The ECG signal can be taken by physionet.org website. With the help of MIT-BIH arrhythmia database we can get normal as well as abnormal patient database. There we have physio toolkit software to extract and read the dat file called rddata.m file. Run this code using MATLAB and extract the ECG .dat signal and give this as an input to your MATLAB code to detect required cardiac arrhythmia.

Database which we have taken for VT and VF are from physionet.org under Creighton University Ventricular tachyarrhythmia Database. This database includes 35, 8 minutes ECG recordings of patient who has experienced VT and VF. In that cu01, cu03 and cu12 are VFIB records and remaining are $\mathrm{VT}$ records. These signals are digitized at $250 \mathrm{~Hz}$ with $1 \mathrm{~V} / \mathrm{mV}$ signal. All signals are passed through LPF with $70 \mathrm{~Hz}$ cut-off frequency and each record contains 127,232 samples. 418.dat is VT signal which has duration of half an hour, and 35.dat, 36.dat and 37.dat are AF recording which have been considered from MIT-BIH arrhythmia database library.

\section{Block diagram implementation using MATLAB:}

One of the simplest recognition of VT, AF and VF is based on the measurement of cardiac rate which can be detected with the help of algorithm. Here we have used Pan and Tompkins algorithm to detect QRS complexes in ECG signal.

Block diagram consist of high pass filter, derivative filter, squaring function, moving window function and R-peak detection block which has been shown below,

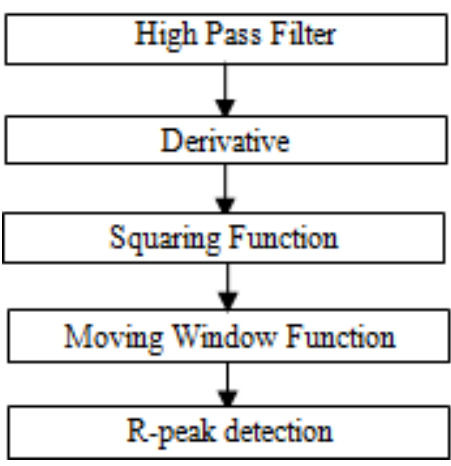

Figure 6: Block diagram representation of Pan and Tompkins algorithm

Each block of Pan and Tompkins algorithm is explained below,

- In the first step of the algorithm, the ECG signal goes through a band-pass filter to attenuate the $\mathrm{P}$ and $\mathrm{T}$ wave of ECG signal, so that to remove low frequency components of the ECG with pass band of $5-12 \mathrm{~Hz}$. Which is the combination of both low pass and high pass filter, LPF with cut-off frequency of $11 \mathrm{~Hz}$ and HPF with cut-off frequency of $5 \mathrm{~Hz}$ is used.

- After filtering $\mathrm{P}$ and $\mathrm{T}$ wave of $\mathrm{ECG}$, the signal is differentiated to amplify the QRS edges.

- The differentiated signal has positive and negative points; it is then squared to get a signal which has only positive peaks.

- The steeper parts which are detected in the differentiated filter are amplified and squaring function will exhibit multiple peaks within duration of single QRS complex. 


\section{International Journal of Science and Research (IJSR) \\ ISSN (Online): 2319-7064}

Index Copernicus Value (2015): 78.96 Impact Factor (2015): 6.391

- To make the signal smoother Pan and Tompkins algorithm uses moving window function.

- Finally, R-peaks are detected and heart rate is calculated.

\section{Results}

\section{1) Normal ECG signal}

The output shown below is the input ECG signal (Normal) which is in .dat, .hea and .atr format. This signal is extracted from Physionet.org website where we can get number of ECG signals.ECG database 100.dat file is shown below,

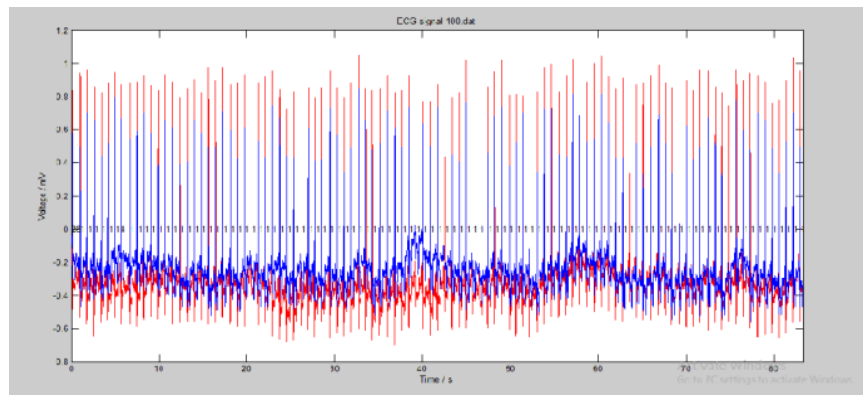

Figure 7: Input ECG 100.dat

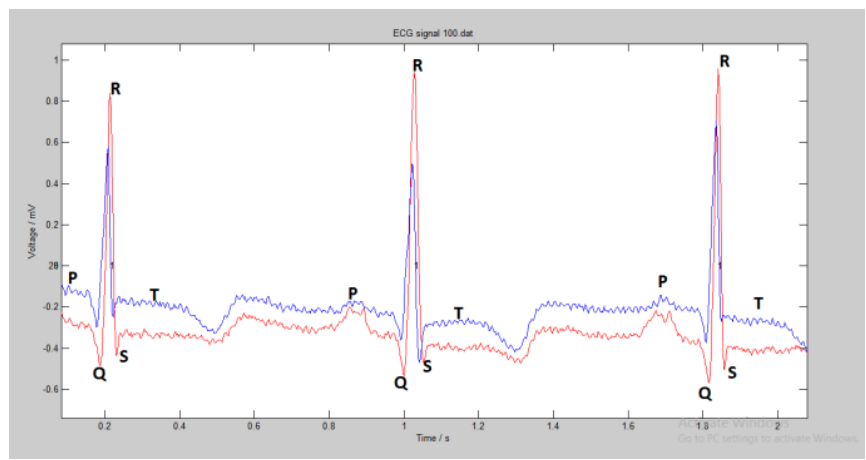

Figure 8: Zoomed in view of 100.dat

\section{Output of normal ECG signal}

The output consist of 4 graphs, first the input signal which has been passed through BPF to remove $\mathrm{P}$ and $\mathrm{T}$ waves of input ECG. After that the signal differentiated, and then it is squared to remove negative peaks of the differentiated signal so that we can get only QRS complexes. By determining Rpeaks we can easily calculate the heart rate. Output graph is shown below, which is of 60s window and detected R-peaks are 74.

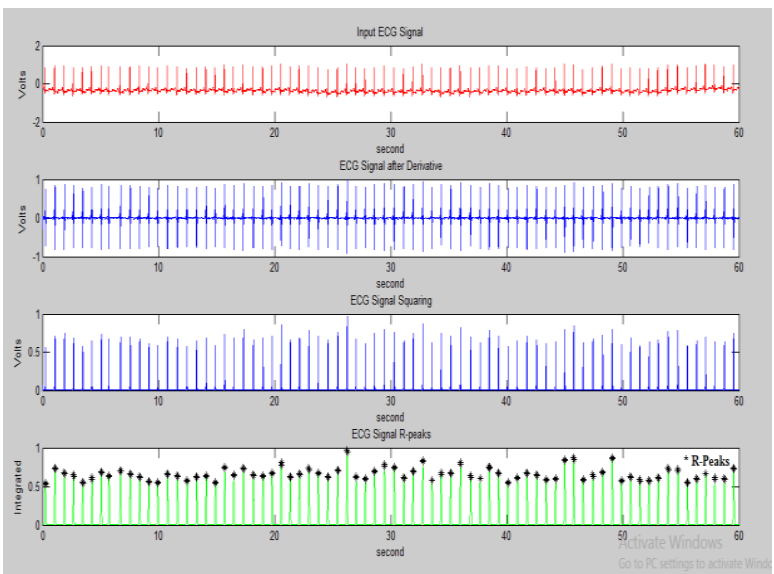

Figure 9: Output of 100.dat ECG

\section{2) Ventricular Tachycardia}

As we taken for input ECG the same procedure repeats for VT signal. Here if we compare input ECG with VT signal the number of R-peaks are more compared with input.

Here, we have considered 418.dat file which represents Ventricular Tachycardia which consists of Time/s along Xaxis and Voltage/mV along $\mathrm{y}$-axis.

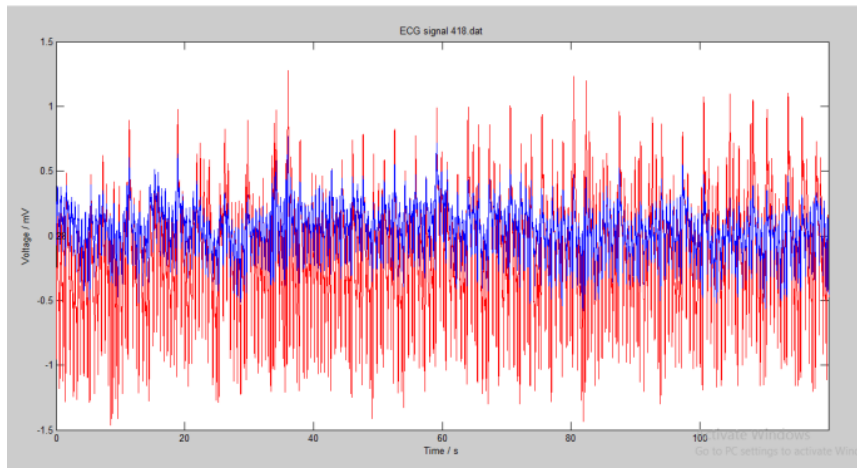

Figure 10: VT input 418.dat ECG

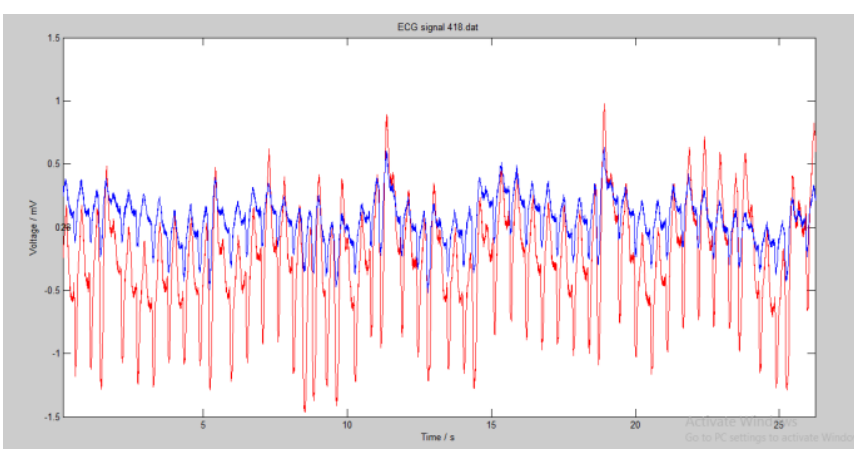

Figure 11: Zoom in of VT input 418.dat

\section{Output of VT detected ECG:}

The output waveform of 20s window which has four plots, in which first plot represents input VT signal, second represents the signal after derivative function has been applied, which has both positive and negative edges, to remove negative edges square the derivate signal to get only positive peaks. Finally in the fourth plot R-peaks are detected.

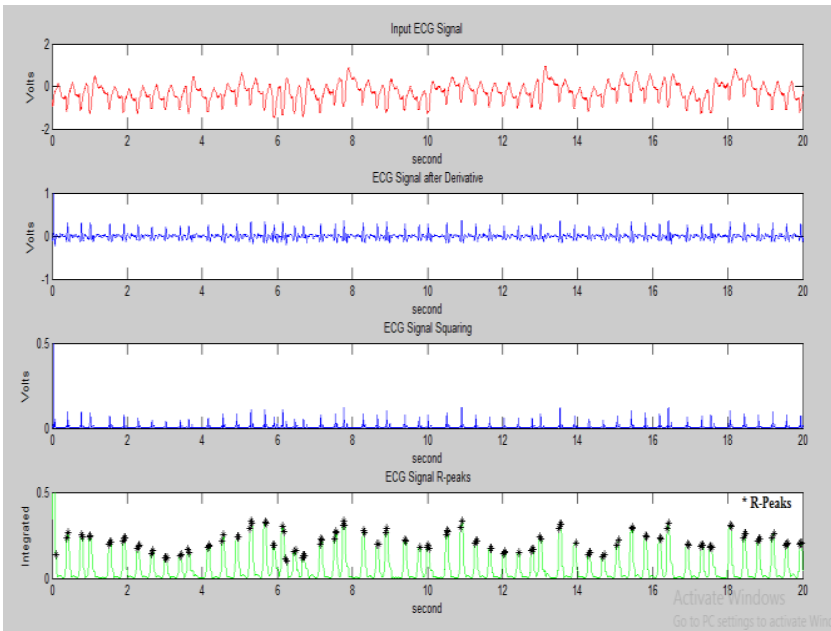

Figure 12: Output of 418.dat VT signal

Volume 6 Issue 7, July 2017 www.ijsr.net 


\section{International Journal of Science and Research (IJSR) \\ ISSN (Online): 2319-7064}

Index Copernicus Value (2015): 78.96 | Impact Factor (2015): 6.391

\section{3) Atrial Fibrillation}

AF and VT are same but here R-peaks are very clear compared to VT and VF. The output of the AF when implemented through algorithm is shown below, We have considered 20s window.
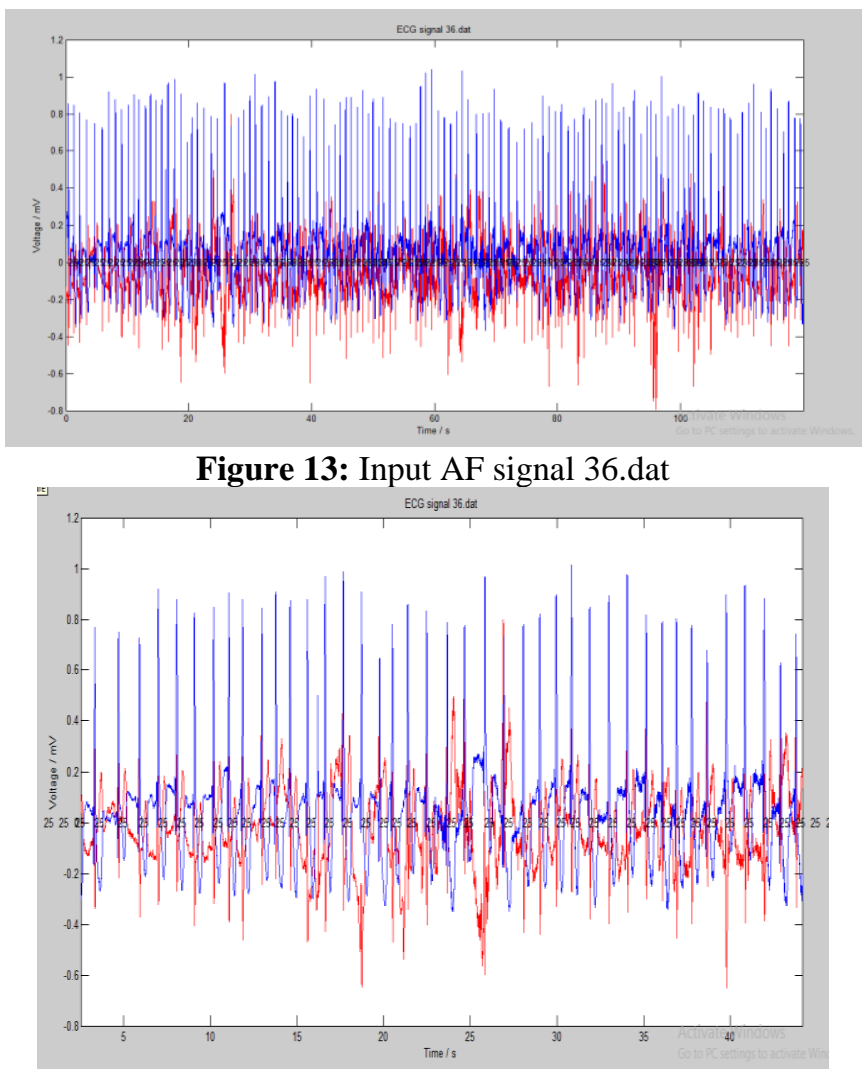

Figure 14: Zoom in of AF input 36.dat

\section{Output of AF detected ECG:}

The output waveform of 20s window which has four plots, in which first plot represents input AF signal, second represents the signal after derivative function has been applied, which has both positive and negative edges, to remove negative edges square the derivate signal to get only positive peaks. Finally in the fourth plot R-peaks are detected.

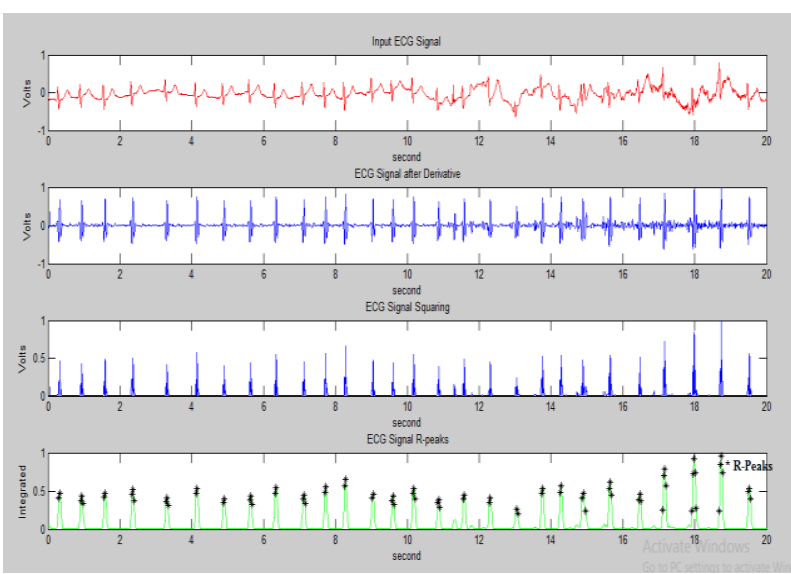

Figure 15: Output of 36.dat AF signal

\section{4) Ventricular Fibrillation}

It is different from VT and AF. The heart rate reaches above 200, the R-peaks are more. But here we cannot recognise Rpeaks properly. The output is shown below,
Here 10s window has been considered and detected R-peak count is 50 and heart rate reaches up to 300 .

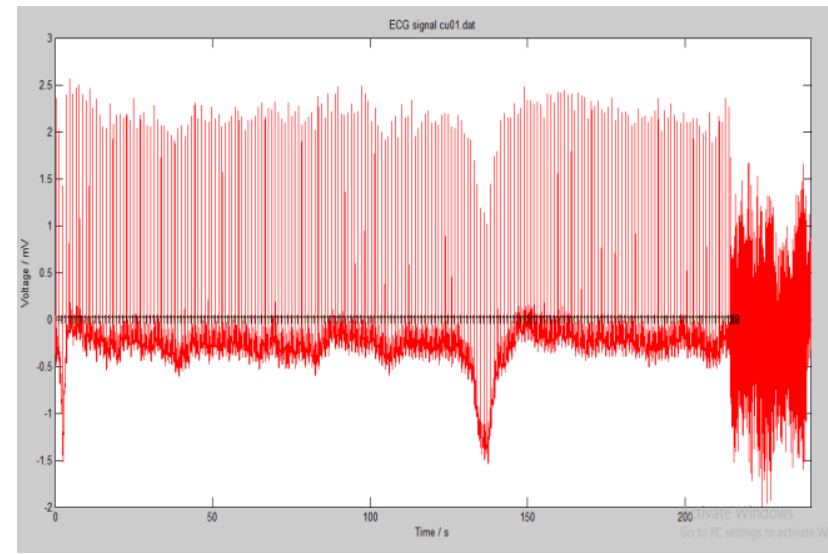

Figure 16: Input of cu01.dat

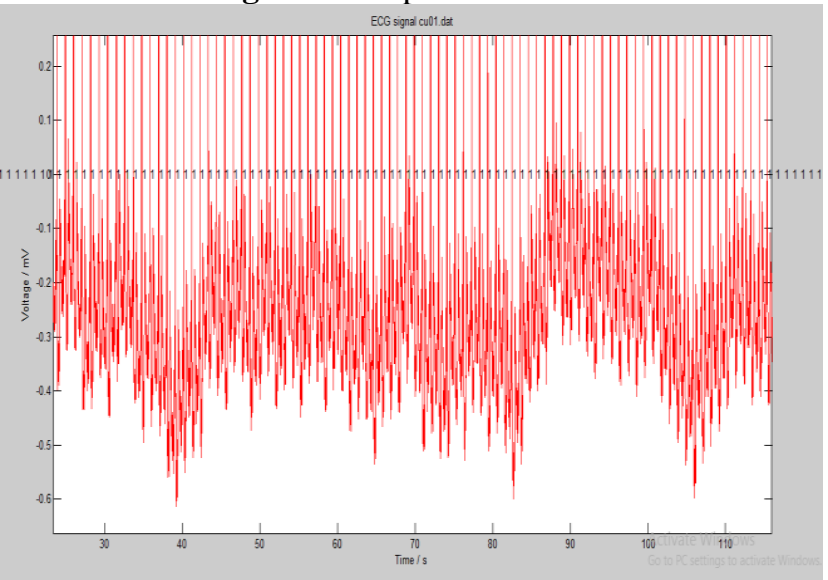

Figure 17: Zoomed in viwe of cu01.dat

\section{Output of VF detected ECG:}

The output waveform of 20s window which has four plots, in which first plot represents input VF signal, second represents the signal after derivative function has been applied, which has both positive and negative edges, to remove negative edges square the derivate signal to get only positive peaks. Finally in the fourth plot R-peaks are detected.

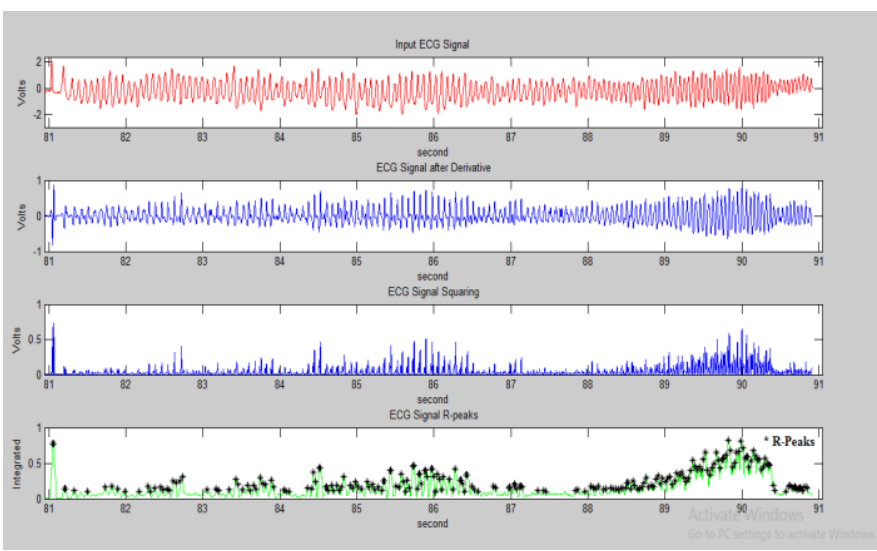

Figure 18: Output of cu01.dat VF signal

\section{Calculation Results}

To calculate number of R-peaks we have to measure overall length of the signal and in that how many R-peaks are there we have calculated by using,

\section{Volume 6 Issue 7, July 2017 www.ijsr.net}




\section{International Journal of Science and Research (IJSR) \\ ISSN (Online): 2319-7064}

Index Copernicus Value (2015): 78.96 | Impact Factor (2015): 6.391

- length (peaks)

Mean RR-interval is calculated using,

- AverageDistance_Peaks

(Calculates average distance between R-peaks)

Using this RR-interval we can calculate frequency as,

- $\mathrm{F}=60 / \mathrm{RR}$

Here we are calculating frequency per minute of the signal so we have considered 60 in the formula.

To calculate total error detection rate, consider the formula:

Total error detection rate $=\left(\frac{\mathrm{FP}+\mathrm{FN}}{\text { Total no. of R-Peaks }}\right) 100$

Where, FP=False Peak Detection

$\mathrm{FN}=$ Failure to Detection

Table 1: R-peaks of ECG signals

\begin{tabular}{|c|c|}
\hline ECG signals taken from physionet.org & Number of R-peaks \\
\hline Normal ECG 100.dat & 74 \\
\hline VT detected ECG 36.dat & 167 \\
\hline AF detected ECG 418.dat & 169 \\
\hline VF detected ECG cu01.dat & 278 \\
\hline
\end{tabular}

Table 2: Mean RR-Interval

\begin{tabular}{|c|c|}
\hline $\begin{array}{c}\text { ECG Signals taken from } \\
\text { physionet.org }\end{array}$ & $\begin{array}{c}\text { Average Distance between } \\
\text { peaks in seconds }\end{array}$ \\
\hline Normal ECG 100.dat & 128.4828 \\
\hline VT detected ECG 36.dat & 107.0144 \\
\hline AF detected ECG 418.dat & 106.4286 \\
\hline VF detected ECG cu01.dat & 64.7225 \\
\hline
\end{tabular}

Table 3: Frequency

\begin{tabular}{|c|c|}
\hline ECG Signals taken from physionet.org & Frequency in $\mathrm{mHz}$ \\
\hline Normal ECG 100.dat & 468.75 \\
\hline VT detected ECG 36.dat & 186.89 \\
\hline AF detected ECG 418.dat & 187.91 \\
\hline VF detected ECG cu01.dat & 309.21 \\
\hline
\end{tabular}

\section{Conclusion}

In this work, the algorithm has been implemented to identify R-peaks to detect VT, AF and VF. The algorithm is called Pan and Tompkins algorithm. This algorithm helps to detect R-peaks in the ECG signal so that we can measure heart rate, total number of R-peaks in the signal, Mean RR-interval and Frequency of the signal. Then based on the heart rate we can detect whether the signal is normal or abnormal. This can be implemented in many of the applications signal processing using cell phone platform, GUI based detection using VHDL etc.

\section{References}

[1] Kritika Bawa, Pooja Sabherwal- "R-Peak Detection by Modified Pan-Tompkins Algorithm", International Journal of Advancements in Research \& Technology, Volume 3, Issue 5, May-2014.

[2] Michał Rospierski, Marcelo Segura, Martín Guzzo, Eduardo Zavalla, Cristian Sisterna and Eric Laciar"Ventricular Fibrillation Detection Algorithm Implemented in a Cell-Phone Platform", Laboratorio de
Electronic Digital (LED) Universidad National de San Juan (UNSJ), San Juan, Argentina.

[3] Priyanka Mundhe, Anand Pathrikal-"Design of an Effective Algorithm for ECG QRS Detection using VHDL", International Journal of Science and Research (IJSR) ISSN (Online): 2319-7064 Impact Factor (2012): 3.358 .

[4] Hussain A. Jaber, AL-Ziarjawey and Ilyas cankaya-"Heart Rate Monitoring and PQRST Detection Based on Graphical User Interface with Matlab", International Journal of Information and Electronics Engineering, Vol. 5, No. 4, July 2015.

Volume 6 Issue 7, July 2017 www.ijsr.net

Licensed Under Creative Commons Attribution CC BY 